\title{
Transformational Electronics - A Powerful Way to Revolutionize Our Information World
}

\author{
Jhonathan P. Rojas ${ }^{\mathrm{a}}$, Galo A. Torres Sevilla ${ }^{\mathrm{a}}$, Mohamed T. Ghoneim ${ }^{\mathrm{a}}$, Aftab M. Hussain ${ }^{\mathrm{a}}$, Sally M. \\ Ahmed $^{\mathrm{a}}$, Joanna M. Nassar ${ }^{\mathrm{a}}$, Rabab R. Bahabry ${ }^{\mathrm{a}}$, Maha Nour ${ }^{\mathrm{a}}$, Arwa T. Kutbee ${ }^{\mathrm{a}}$, Ernesto Byas ${ }^{\mathrm{a}}$, \\ Bidoor Al-Saif ${ }^{\mathrm{a}}$, Amal M. Alamri ${ }^{\mathrm{a}}$, and Muhammad M. Hussain ${ }^{\mathrm{a} *}$ \\ antegrated Nanotechnology Lab, Electrical Engineering Department, Computer, Electrical and \\ Mathematical Sciences and Engineering, 4700 King Abdullah University of Science and \\ Technology, Thuwal 23955, Saudi Arabia \\ *Corresponding Author's E-mail: muhammadmustafa.hussain@kaust.edu.sa
}

\begin{abstract}
With the emergence of cloud computation, we are facing the rising waves of big data. It is our time to leverage such opportunity by increasing data usage both by man and machine. We need ultra-mobile computation with high data processing speed, ultra-large memory, energy efficiency and multi-functionality. Additionally, we have to deploy energy-efficient multi-functional 3D ICs for robust cyber-physical system establishment. To achieve such lofty goals we have to mimic human brain, which is inarguably the world's most powerful and energy efficient computer. Brain's cortex has folded architecture to increase surface area in an ultra-compact space to contain its neuron and synapses. Therefore, it is imperative to overcome two integration challenges: (i) finding out a low-cost 3D IC fabrication process and (ii) foldable substrates creation with ultra-large-scale-integration of high performance energy efficient electronics. Hence, we show a low-cost generic batch process based on trench-protect-peel-recycle to fabricate rigid and flexible 3D ICs as well as high performance flexible electronics. As of today we have made every single component to make a fully flexible computer including non-planar state-of-the-art FinFETs. Additionally we have demonstrated various solid-state memory, movable MEMS devices, energy harvesting and storage components. To show the versatility of our process, we have extended our process towards other inorganic semiconductor substrates such as silicon germanium and III-V materials. Finally, we report first ever fully flexible programmable silicon based microprocessor towards foldable brain computation and wirelessly programmable stretchable and flexible thermal patch for pain management for smart bionics.
\end{abstract}

Keywords: Bulk silicon (100), flexible electronics, 3D ICs, brain computation, flexible microprocessor

\section{INTRODUCTION}

In the world of rising usage of portable electronics, consumers are constantly looking for a great variety of functionalities in their electronic gadgets such as high performance, multi-functionality, interactivity, affordability, ultramobility, light weight and energy efficiency, which can also be seen as long battery lifetime. In response to this demand, industry is focusing their effort to develop new technologies and introduce exciting novel properties such flexibility or bendability. Flexible organic-based screens have been shown in the past and they promise a big amount of original devices for applications in smart bionics, integrated cyber-physical systems and wearable electronics. The later is a great example on how mechanical flexibility can be a game changer characteristic that allows systems to be ubiquitous and easy to deploy. Such systems must be worn on the user's body for an extended period of time, significantly enhancing the user's experience in a different variety of ways for application in medical and healthcare, fitness and wellness, entertainment, industrial and military. We are looking at a growing market that will be first boosted up by social media and entertainment, featuring products such as smart clothing, watches and glasses, then followed by medical and wellness, with products such as health monitors and drug delivery systems. In order to implement any of those products, scientist, engineers and visionaries need to develop new 3D-based flexible architectures with high performance data capabilities that can communicate wirelessly and through a cloud computing system to achieve the required multifunctionality and interactivity as shown in figure 1 .

Micro- and Nanotechnology Sensors, Systems, and Applications VI, edited by Thomas George,

M. Saif Islam, Achyut K. Dutta, Proc. of SPIE Vol. 9083, 90831K · C 2014 SPIE

CCC code: $0277-786 X / 14 / \$ 18 \cdot$ doi: $10.1117 / 12.2050103$ 


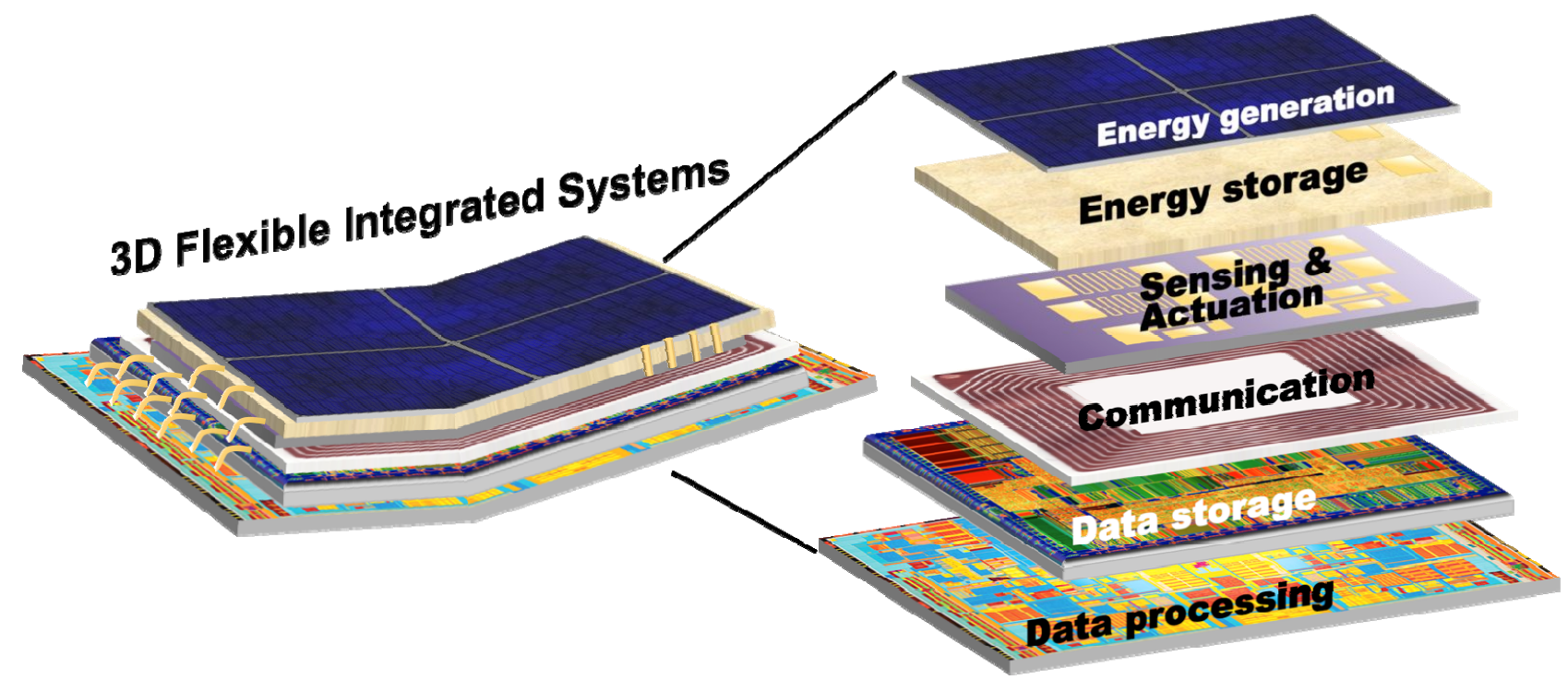

Figure 1. Schematic showing a flexible and 3-dimentional stack architecture integrated system with detailed components for applications in wearable electronics and such.

Furthermore, if we follow the most powerful and energy-efficient computing system, the human brain, we realize that the only way to accommodate such vast amount of single neurons (transistors) and synapses (interconnects) in a small area is to develop 3-dimentional foldable and bendable architecture based electronic systems with ultra-large-scaleintegration to obtain the highest performance and best area-efficiency.

Nevertheless, the current traditionally rigid electronic components cannot yet be used on compliant, complex, wrinkled, wavy and foldable geometries and surfaces as required for different applications. The need to offer effectively flexible, stretchable and compliant electronics, featuring ultra large scale integration (ULSI) and nano-metric critical dimensions, in order to achieve the same level of high performance of current silicon-based integrated circuits, has become a lofty goal for the semiconductor industry as well as many research institutions and groups around the globe. Academics have played an important role in the development of innovative technologies, strategies, and engineering tools to back-up the further advancement of this raising field. On the one hand, organics have played an important role in areas such as displays and sensor arrays [1-3], and they show promise for implementation at big scale electronics, also know as macroelectronics, due to their cost effectiveness and simplicity. Nonetheless, for applications where high performance is needed, this technology is not suitable due to the inherent low mobility of organic materials. Furthermore, thermal budget constrains also hinders their applications and compatibility with other processes usually performed during stateof-the-art device fabrication.

On the other hand we have inorganic materials, leaded by silicon, displaying outstanding electrical properties. More than five decades of silicon-based electronics' evolution has allowed the current data processing and storage capabilities, which at the same time are the basis of today's information era. A density up to 5 billion transistors in an area as small as $2 \mathrm{~cm} \times 2 \mathrm{~cm}$, is nowadays only possible through the development of photolithographic techniques and several other micro- and nano-fabrication processes, which have been highly optimized for semiconductor materials, especially silicon. On the mechanics perspective, semiconducting inorganic materials tend to be actually brittle and not easily bendable. Looking at the definition of flexural rigidity $(D)$ a work-around this issue has been identified. Rigidity or stiffness of a structure is the resistance while it is being bent as defined in the following equation for a rectangular geometry [4],

$$
D=\frac{E \cdot w \cdot t^{3}}{12}
$$

Where $E$ is the Young's modulus, $t$ the thickness and $w$ the width. As can be inferred, the rigidity of the structure highly depends on the thickness, reducing drastically as the thickness decreases. Therefore by reducing the thickness of the 
semiconducting inorganic material we can achieve high bendability, although handling becomes a different new issue. Several research groups have devised diverse strategies to go around this problem. John Rogers' group and others have implemented a transfer printing technology, which uses released ultra-thin silicon sheets (from unconventional siliconon-insulator (SOI) and Si (111) substrates) that are then transferred and printed on polymeric substrates as mechanical support [5-10]. Several interesting and inventive systems have been demonstrated using such technology for applications in medical, imaging, sensing and others where irregular shapes are needed [11-13]. Such transfer process can achieve alignment in order of micrometers, which might be enough for some specific applications as the ones mentioned earlier, but it is still far away from the nano-metric requirements of today's high performing computation. Furthermore, the unconventional substrates used to release the ultra-thin sheet of silicon can be up to five times more expensive than the standard silicon (100) wafers.

A different approach relies on chemical anodization processes to create porous silicon layers of different degrees of porosity to create a void inside the wafer after a very high thermal budget process, on top of which a new layer of epitaxial grown silicon will host electronic devices and circuits. Lastly the thin layer of hanging silicon can be picked and placed on top of a polymer substrate $[14,15]$. Functional circuitries have been demonstrated although the set of processes used during the whole procedure can be very expensive, complex and non-standard. Another option has recently been explored where spalling or exfoliation of a sheet of silicon can be obtained from a wafer, which has been used to show device and circuitry functionality of different complexities, but it involves a complex process itself, which uses a metal stressor and fracture controlled peeling of the substrate's top portion, but has a limitation of the minimum released sheet's thickness to tens of micrometers [16-18]. Additionally, both of these processes yield in opaque flexible electronics with limited bendability.

With the aim of overcoming all these issues, we have developed and demonstrated a low-cost generic batch process based on trench-protected release that allows us to transform and extend any traditionally rigid but high-performing electronics into a flexible and semitransparent platform for systems that involve wavy and irregular surfaces. Our process is versatile as it encompasses from bulk mono-crystalline silicon (100) to dielectric materials, and from silicon germanium ( $\mathrm{SiGe}$ ) to III-V inorganic semiconductor substrates. As of today we have demonstrated: metal-oxidesemiconductor capacitors (MOSCAPs) and field effect transistors (MOSFETs), metal-insulator-metal capacitors (MIMCAPs), 3D-fin field effect transistors (FinFET), memory, micro electro-mechanical systems (MEMS), energy harvesting and storage devices [19-26]. Additionally we for the first time show a pathway for a fully flexible programmable silicon microprocessor and a wirelessly programmable stretchable and flexible thermal patch for pain management. Both are steps forward toward smart-bionics, ubiquitous wearable electronics and foldable braincomputation.

\section{FABRICATION PROCESS DESCRIPTION}

As denoted previously our process can be used to release several kinds of materials and as such, some considerations need to be taken into account for each case. Using a standard silicon wafer as starting point and carrier substrate, we are able to release a sheet of certain materials deposited on top depending upon the selectivity of the material to be released with respect to the silicon carrier wafer. First, in case of dielectric materials, such as silicon dioxide $\left(\mathrm{SiO}_{2}\right)$, which are mostly selective to silicon, they can be directly deposited on top of the carrier wafer, and then, through an array of photolithography-defined etching holes, the silicon below the dielectric can be removed releasing the top layer. In the case of silicon dioxide $\left(\mathrm{SiO}_{2}\right)$, silicon nitride $\left(\mathrm{Si}_{3} \mathrm{~N}_{4}\right)$, aluminum oxide $\left(\mathrm{Al}_{2} \mathrm{O}_{3}\right)$ and others, xenon difluoride $\left(\mathrm{XeF}_{2}\right)$ can be used to chemically remove the silicon underneath the dielectric material (Figure 2a).

Secondly, materials not selective to silicon, such polycrystalline and amorphous silicon, an additional "sacrificial" layer needs to be introduced, which then can be selectively removed to release the layer on top. Plasma enhanced chemical vapor deposition (PECVD) based $\mathrm{SiO}_{2}$ can be used as sacrificial layer, on top of which the material of interest can be deposited. Again, an array of etching holes is used to effectively remove the underlying sacrificial layer. In the case of $\mathrm{SiO}_{2}$ vapor hydrofluoric acid (VHF) can be used to selectively and isotropically etch away the oxide layer without affecting the layer on top (Figure 2b).

The third case consists of mono-crystalline silicon itself. We have relied on the lowest-cost, most widely used substrate in the semiconductor industry, namely silicon (100) wafer. Similarly to the procedures described earlier, we make use of an array of releasing or etching holes, deeply etched in the wafer to a desire depth, to remove an inner layer of the substrate without affecting the top most portion of the wafer where high performing devices have been fabricated. In 
order to achieve this, we have introduced the formation of spacer-like wall protection to shield the silicon sidewalls of the etching holes from the chemically reactive gas used to remove the inner silicon layer, in our case $\mathrm{XeF}_{2}$ (Figure 2c). We have selected this etchant mainly because of its high selectivity to diverse materials (several polymers, metals and dielectrics commonly used during integrated circuits (IC) fabrication), making it thus compatible with complementary metal oxide semiconductor (CMOS) post-processing. Furthermore, the remaining bottom portion of the substrate can be actually recycled to obtain several sheets or fabrics with devices for a better cost-effectiveness use of the material [27].
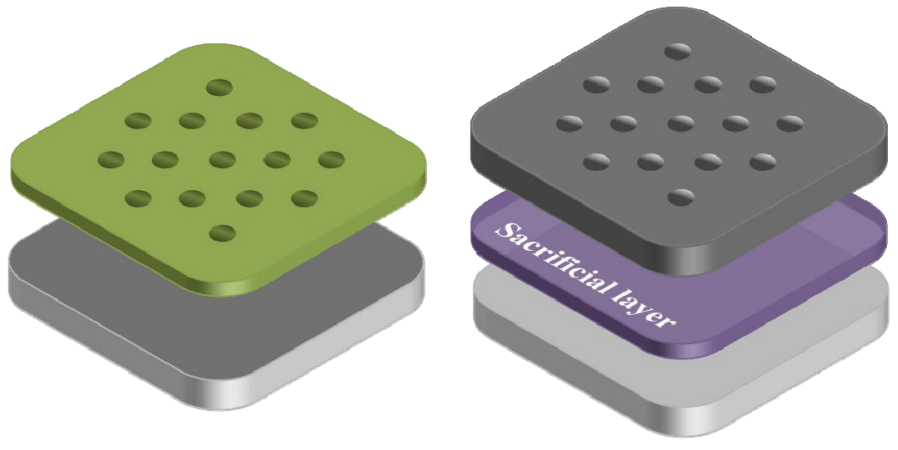

a. Selective materials to silicon

b. Non-selective materials to silicon

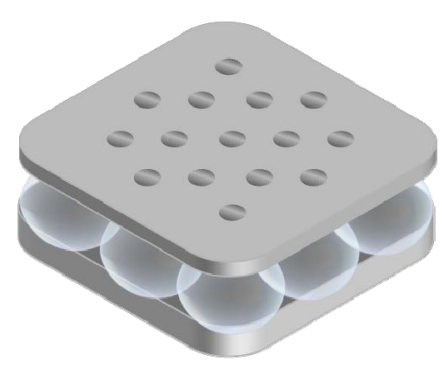

c. Mono-crystalline silicon

Figure 2. Schematics for releasing of different materials depending on selectivity to silicon.

The fourth case is related to the release of alternative semiconductors to silicon such as III-V family or silicon germanium, which are attractive thanks to their unique electrical properties such as higher mobilities for ultra high performance applications or relevance to optoelectronics industry. In the case of semiconductor materials being deposited on top of a silicon substrate through hetero-epitaxial growth (using molecular beam epitaxy (MBE), metal organic chemical vapor deposition (MOCVD) or others), the same method described in the third case can be used to release a thin layer of silicon with the grown semiconductor on the very top. Additionally, if the substrate itself is solely made of the III-V semiconductor, the third method can be also used given that anisotropic and isotropic etching techniques can be done, and there exist an adequate combination of selective etchant and sidewall-protection layer. Lastly, some cases of substrates involve multilayer of semiconductors grown on top of each other, in which case a similar technique as in the first case can be implemented provided a suitable selective etchant can be found, e.g. selective removal of aluminum arsenide (AlAs) layer to release a top gallium arsenide (GaAs) sheet.

Finally, it its worth to point our that the thickness of the released material can be controlled by the amount of material deposited in the case of the first and second approach, whereas in the third case, the etching-holes density and depth defines the final thickness of the released silicon fabric. Moreover, the released fabrics are optically semitransparent due to the presence of the array of etching holes. Specific processing details of the previous procedures can be found in our prior works $[26,28]$.

\section{RESULTS AND DISCUSSION}

To demonstrate the adaptability, usefulness and convenience of our process, we have fabricated and performed a detailed characterization of several electronic components and devices, not limited to logic but also in energy and MEMS applications. Figure 3 summarizes such device demonstrations and elucidates how they can work together for the future development of 3D-based flexible architectures with high performance data processing and storage and advance sensing capabilities that can communicate wirelessly and be self-sustainable.

The first device demonstration consisted on MOSCAPs fabricated both with a release-first/device-second and devicefirst/release-second. In the first case, a thin silicon fabric was first released as described earlier and next, capacitors were fabricated on top while the fabric was still attached to the substrate for easy handling. In the second approach, devicefirst/release-second, capacitors were first fabricated, etching holes included, and then the process described in the 
previous section (third case) was performed to release the silicon fabric with devices. We have selected MOSCAP as first device demonstration as they are not only fundamental building blocks for CMOS and MEMS technologies but also are one of the largest devices in their families, which shows that much smaller devices, as standard transistors, can be easily implemented without being affected by the presence of holes. Moreover, our devices featured the state-of-the-art material selection with high-k/metal gate stack to improve performance at lower leakage for better energy efficiency [19, $20]$.

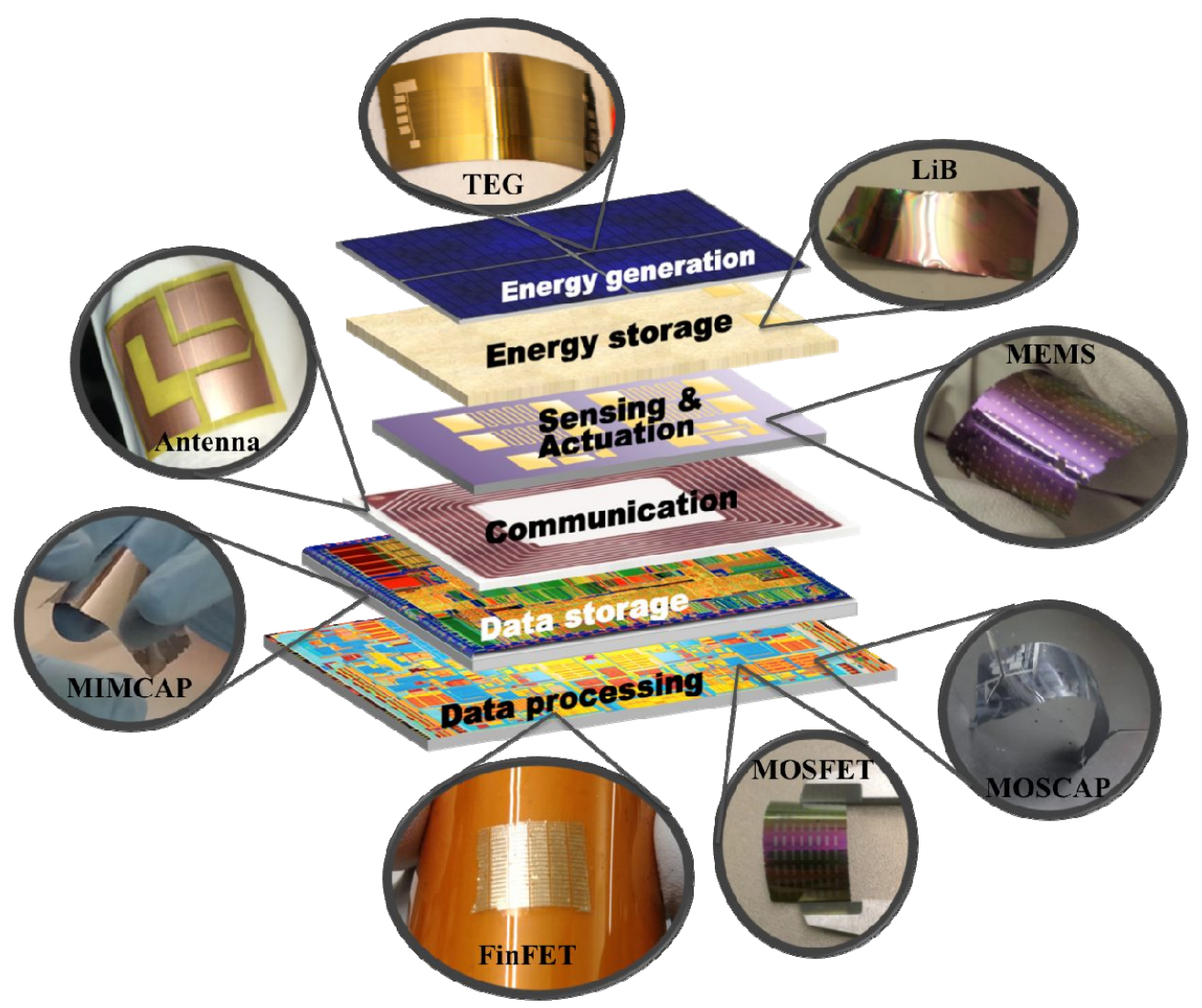

Figure 3. Schematics of components for a comprehensive and ubiquitous systems and its respective real photographs of devices developed on our flexible silicon fabric.

Our second device implementation was the fabrication of metal-insulator-metal (MIM) capacitors, key components of dynamic random access memories (DRAM). The approach was release-first/device-second and for the first time in flexible electronics field, we performed an electrical reliability study that indicated no significant difference in performance and showed an improvement in lifetime projections after release [22]. In addition, the fabricated devices displayed increased capacitance per unit planar area up to 2 times compared to the un-released devices. The reason behind this behavior is the additive advantage of the particular non-planar geometry on account of the presence of the etching holes array, which increases the effective active area. Besides, this additional area arrangement of the released flexible devices did not seem to increase the static leakage current compared to their un-released bulky counterparts that used the same chip area [29].

Third, we fabricated metal-oxide-semiconductor field effect transistors (MOSFETs) with the device-first/release-second approach. Again we used the most advanced gate material stack and improved contact resistance due to nickel silicide (NiSi) implementation. These improvements led to an ultra steep current-voltage (I-V) characteristic, only $20 \mathrm{mV}$ above the theoretical limit imposed by the thermionic nature of the electrons' transport in CMOS transistors. This achievement is of big importance to be able to reduce the applied voltage and thus achieve ultra-low power consumption in such 
flexible platforms. In terms of flexibility we achieved a remarkable small minimum bending radius and showed that no electrical parameter used to characterize the device varied significantly even under high strain conditions [21].

We have also demonstrated for the first time movable MEMS devices on a flexible substrate. Using the devicefirst/release-second approach we fabricated two-armed thermal flexure actuators demonstrating functionality without degradation after release, which suggests how our process can be used to expand the horizon of flexible electronics into MEMS world devices [24].

Additionally, we have also ventured into the arena of energy generation and storage. In the world of mobile and ubiquitous wearable electronics, being able to provide energy at any given moment and for long periods of time is as important or more than being able to place your device on any irregular surface. That is why we have implemented both a thermoelectric generator as energy scavenging system and a lithium ion battery as energy storage system. The first one was fabricated following the device-first/release-second approach and showed a power generation up to 30 times higher than the same system fabricated on standard rigid and thick silicon wafer [25]. On the other hand, the battery was fabricated after the silicon fabric was released achieving a remarkable minimum bending radius and competitive capacity $[26,27]$.

Furthermore, we have made an important breakthrough in the field of flexible electronics by demonstrating the first ever 3-dimentional-architecture transistor just recently implemented by industry in their ultra high performance and energy efficient microprocessors. The FinFET promises an improved electrostatic control, superior levels of scalability and lower leakage currents compared with the standard planar transistor. Therefore we have expanded of transformational process to release the fin structures from an SOI wafer and then transfer it into a polymide substrate as flexible mechanical support. The released devices showed competitive electrical behavior and outstanding bendability relying only on mature microfabrication processes. We believe this process sets a major step towards the expansion of ultramobile high performance flexible electronics with the ultra-area-efficiency and folding capability of the human brain [23].

Following this same line of brain computation, we report the pathway for the development of the first ever fully flexible programmable silicon based microprocessor. It consists of a 4 bits ripple carry adder with four full adders, which each carry is input of the next adder. We strongly believe this step will set the path towards foldable brain computation.

We are currently also working on the development of wireless systems with flexible antennas fabricated both on top of polymeric substrates, as well as on our silicon fabric. Such antennas will enable a multitude of applications for remote control of cyber-physical systems, wearable electronics and smart bionics. Specific examples of such systems are comprehensive health monitors of several vital signs for both prevention and in-situ treatment of different diseases, and ultra-sensitive electronic skin for remote medical treatment based on augmented reality.

Lastly, in order to increase the applicability of our process, we have also implemented a not only flexible but also stretchable thermal patch system for pain management, which can be wirelessly programmable. The idea was to make use of the extreme bendability and stretch-ability of polymers to be the mechanical support of conductive lines that will heat to a certain temperature upon an applied voltage. Such voltage, and therefore temperature, can be controlled wirelessly via your smartphone, which takes us one step closer to real ubiquitous wearable electronics and smart bionic systems.

\section{CONCLUSION}

We have developed a revolutionary process that allows transforming any kind of traditionally rigid electronics into a flexible and semitransparent platform for rapid deployment of ultra-mobile, high-performing, multi-functional and interactive electronic gadgets. Our process offers: (i) ultra-large-scale-integration capability; (ii) usability of advanced

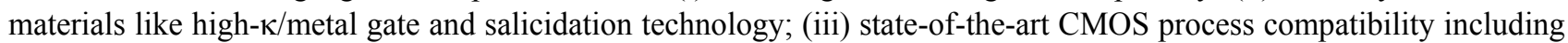
high thermal budget processes; (iv) integration of vertically aligned complex non-planar 3D geometric devices such as nanowire FETs and FinFETs; (v) capability of recycling of the silicon substrate for a better cost-effectiveness use of the material. We have successfully used our technique to demonstrate several logic components as well as MEMS, energy harvesting and storage devices, and even transform new materials such III-V and SiGe. We believe this technique can leverage the rise of flexible inorganic electronics involving high performance computation and effective communication systems with sensors, displays and power autonomy for implementation in wearable electronics, brain computation and smart bionics. 


\section{REFERENCES}

[1] R. H. Reuss, B. R. Chalamala, A. Moussessian et al., "Macroelectronics: Perspectives on Technology and Applications," Proceedings of the IEEE, 93(7), 1239-1256 (2005).

[2] H. Eun-Soo, S. Jung-hoon, and K. Yong-Jun, "A Polymer-Based Flexible Tactile Sensor for Both Normal and Shear Load Detections and Its Application for Robotics," Microelectromechanical Systems, Journal of, 16(3), 556563 (2007).

[3] M. Y. Cheng, C. M. Tsao, Y. Z. Lai et al., "The development of a highly twistable tactile sensing array with stretchable helical electrodes," Sensors and Actuators A: Physical, 166(2), 226-233 (2011).

[4] A. Maceri, [Theory of Elasticity] Springer Berlin Heidelberg, Rome, 356-426 (2010).

[5] A. Carlson, A. M. Bowen, Y. Huang et al., "Transfer Printing Techniques for Materials Assembly and Micro/Nanodevice Fabrication," Advanced Materials, 24(39), 5284-5318 (2012).

[6] H.-J. Chung, T.-i. Kim, H.-S. Kim et al., "Fabrication of Releasable Single-Crystal Silicon-Metal Oxide FieldEffect Devices and Their Deterministic Assembly on Foreign Substrates," Advanced Functional Materials, 21(16), 3029-3036 (2011).

[7] S.-I. Park, J.-H. Ahn, X. Feng et al., "Theoretical and Experimental Studies of Bending of Inorganic Electronic Materials on Plastic Substrates," Advanced Functional Materials, 18(18), 2673-2684 (2008).

[8] J.-H. Ahn, H.-S. Kim, E. Menard et al., "Bendable integrated circuits on plastic substrates by use of printed ribbons of single-crystalline silicon," Applied Physics Letters, 90(21), 213501-213501-3 (2007).

[9] H.-S. Kim, S. M. Won, Y.-G. Ha et al., "Self-assembled nanodielectrics and silicon nanomembranes for low voltage, flexible transistors, and logic gates on plastic substrates," Applied Physics Letters, 95(18), 183504183504-3 (2009).

[10] T.-i. Kim, Y. Hwan Jung, H.-J. Chung et al., "Deterministic assembly of releasable single crystal silicon-metal oxide field-effect devices formed from bulk wafers," Applied Physics Letters, 102(18), 182104-182104-4 (2013).

[11] H.-J. Chung, M. S. Sulkin, J.-S. Kim et al., "Stretchable, Multiplexed pH Sensors With Demonstrations on Rabbit and Human Hearts Undergoing Ischemia," Advanced Healthcare Materials, 3(1), 59-68 (2014).

[12] D.-H. Kim, J. Viventi, J. J. Amsden et al., "Dissolvable films of silk fibroin for ultrathin conformal bio-integrated electronics," Nat Mater, 9(6), 511-517 (2010).

[13] R. C. Webb, A. P. Bonifas, A. Behnaz et al., "Ultrathin conformal devices for precise and continuous thermal characterization of human skin," Nat Mater, 12(10), 938-944 (2013).

[14] J. N. Burghartz, W. Appel, H. D. Rempp et al., "A New Fabrication and Assembly Process for Ultrathin Chips," Electron Devices, IEEE Transactions on, 56(2), 321-327 (2009).

[15] H. Sanda, J. McVittie, M. Koto et al., "Fabrication and characterization of CMOSFETs on porous silicon for novel device layer transfer." 679-682.

[16] D. Shahrjerdi, and S. W. Bedell, "Extremely Flexible Nanoscale Ultrathin Body Silicon Integrated Circuits on Plastic," Nano Letters, 13(1), 315-320 (2012).

[17] D. Shahrjerdi, S. W. Bedell, A. Khakifirooz et al., "Advanced flexible CMOS integrated circuits on plastic enabled by controlled spalling technology." 5.1.1-5.1.4.

[18] Y. Zhai, L. Mathew, R. Rao et al., "High-Performance Flexible Thin-Film Transistors Exfoliated from Bulk Wafer," Nano Letters, 12(11), 5609-5615 (2012).

[19] J. P. Rojas, and M. M. Hussain, "Flexible semi-transparent silicon (100) fabric with high-k/metal gate devices," physica status solidi (RRL) - Rapid Research Letters, 7(3), 187-191 (2013).

[20] J. P. Rojas, G. T. Sevilla, and M. M. Hussain, "Structural and electrical characteristics of high-k/metal gate metal oxide semiconductor capacitors fabricated on flexible, semi-transparent silicon (100) fabric," Applied Physics Letters, 102(6), 064102-064102-4 (2013).

[21] J. P. Rojas, G. A. Torres Sevilla, and M. M. Hussain, "Can We Build a Truly High Performance Computer Which is Flexible and Transparent?," Sci. Rep., 3, (2013).

[22] J. P. Rojas, M. T. Ghoneim, C. D. Young et al., "Flexible High-k/Metal Gate Metal/Insulator/Metal Capacitors on Silicon (100) Fabric," Electron Devices, IEEE Transactions on, 60(10), 3305-3309 (2013).

[23] G. A. T. Sevilla, J. P. Rojas, H. M. Fahad et al., "Flexible and Transparent Silicon-on-Polymer Based Sub-20 nm Non-planar 3D FinFET for Brain-Architecture Inspired Computation," Advanced Materials, n/a-n/a (2014).

[24] S. M. Ahmed, A. M. Hussain, J. P. Rojas et al., "Solid state MEMS devices on flexible and semi-transparent silicon (100) platform." 548-551. 
[25] G. A. T. Sevilla, S. B. Inayat, J. P. Rojas et al., "Flexible and Semi-Transparent Thermoelectric Energy Harvesters from Low Cost Bulk Silicon (100)," Small, 9(23), 3916-3921 (2013).

[26] J. P. Rojas, G. A. Torres Sevilla, M. T. Ghoneim et al., "Transformational Silicon Electronics," ACS Nano, 8(2), 1468-1474 (2014).

[27] G. T. Sevilla, J. P. Rojas, S. Ahmed et al., "Silicon fabric for multi-functional applications." 2636-2639.

[28] J. P. Rojas, A. Syed, and M. M. Hussain, "Mechanically flexible optically transparent porous mono-crystalline silicon substrate." 281-284.

[29] M. T. Ghoneim, J. P. Rojas, A. M. Hussain et al., "Additive advantage in characteristics of MIMCAPs on flexible silicon (100) fabric with release-first process," physica status solidi (RRL) - Rapid Research Letters, 8(2), 163-166 (2014). 